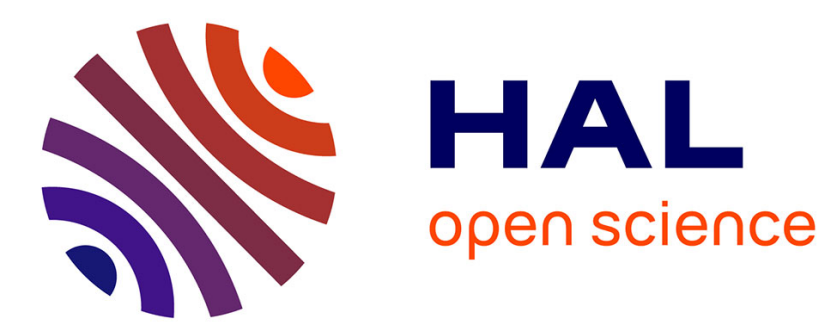

\title{
Réforme politique et éducation : un dialogue Godwin-Helvétius sur perfectibilité
}

Sophie Audidière

\section{To cite this version:}

Sophie Audidière. Réforme politique et éducation: un dialogue Godwin-Helvétius sur perfectibilité. Dialogue: Canadian Philosophical Review / Revue canadienne de philosophie, 2007, 46 (2), pp.287309. 10.1017/S001221730000175X . halshs-02077794

\section{HAL Id: halshs-02077794 https://shs.hal.science/halshs-02077794}

Submitted on 31 Mar 2019

HAL is a multi-disciplinary open access archive for the deposit and dissemination of scientific research documents, whether they are published or not. The documents may come from teaching and research institutions in France or abroad, or from public or private research centers.
L'archive ouverte pluridisciplinaire HAL, est destinée au dépôt et à la diffusion de documents scientifiques de niveau recherche, publiés ou non, émanant des établissements d'enseignement et de recherche français ou étrangers, des laboratoires publics ou privés. 


\section{Réforme politique et éducation : un Godwin-Helvétius sur la perfectibilité}

SOPHIE AUDIDIÈRE Chaire UNESCO-UQAM d'Étude des Fondements philosophiques de la Justice et de la Société démocratique

\section{XLVI (2007), 287-309}

RÉSUMÉ : Cet article examine le rapport de Godwin (1756-1836), écrivain anglais «jacobin», auteur de Political Justice (1793), aux philosophes français, Helvétius en particulier. Partisans d'une politisation de la théorie de la connaissance plutôt que d'une intellectualisation de la politique, ils interrogent sans cesse, via la question de l'éducation, les liens du pouvoir politique, de l'histoire de l'entendement humain et de la science morale. Après les massacres de septembre (1792), la position de Godwin évolue considérablement et met en scène la perfectibilité. Le différend final marque la distinction entre un courant des Lumières françaises, réformatrices et étatistes, et un courant des Lumières anglaises, aux sources religieuses dissidentes ou réformées, porteuses, elles, de l'idée de perfectibilité.

ABSTRACT: This article analyzes the relation between Godwin (1756-1836), Republican and author of Politcal Justice (1793), and French philosophers, particularly Helvétius. Both Godwin and Helvétius were in favour of a political understanding of the theory of knowledge as opposed to an intellectual treatment of policy. They continually questioned the links between policy, history of the human understanding, and moral science from the perspective of the question of education. After the September Massacres (1792), Godwin's thought changed radically and began to revolve around the notion of perfectibility. The final disagreement marked a distinction between French reformers, who advocated state control, and English writers, influenced by dissent and reformation, who held the idea of perfectibility.

On compte en général Godwin (1756-1836) parmi les écrivains anglais dits jacobins, qui prirent parti pour la Révolution française, dans un premier temps du moins, envoyèrent en France des manifestations de soutien, des projets politiques portant sur le suffrage ou la Constitution, etc. Les événements de France leur servirent également de matériaux pour élaborer la critique de leur propre système politique monarchique. En 1793, lorsque paraît la première édition de Enquiry Concerning Political Justice and Its Influence on Morals and Happiness, Godwin prend acte des révolutions d'Amérique et de France, les approuve, et se revendique dans la préface de ce qu'il nomme le «républicanisme» de philosophes français des Lumières : d'Holbach, Rousseau, Helvétius. Or au fil de l'œuvre, cette filiation est tantôt affirmée, tantôt désavouée par l'auteur lui-même, directement ou par des positions philosophiques. Nous souhaitons examiner ici ce qu'il en est précisément de ce rapport de Godwin aux philosophes français cités, et à Helvétius en particulier. En effet, Godwin continue à se confronter à la pensée d'Helvétius jusqu'en 1831. L'évaluation de cette source française dans la philosophie de Godwin a déjà été tentée, mais avec des résultats contradictoires; en outre, il semble encore nécessaire d'en identifier l'enjeu, au travers des stratégies explicites de filiation qui ne sont pas toujours des filiations ou des oppositions doctrinales aussi immédiates que les auteurs eux-mêmes l'admettent. Il nous semble qu'en suivant plus particulièrement la question de la perfectibilité dans sa dimension politique, on peut faire apparaître des tendances nationales divergentes au sein de la philosophie éclairée et républicaine. C'est là l'enjeu de l'évaluation de la place de certains représentants radicaux des Lumières françaises (au premier rang desquels se trouve Helvétius), dans l'Angleterre de la fin du siècle, et plus particulièrement dans le milieu intellectuel qui est celui de Godwin : les protestants dissidents qui ont inspiré et s'inspirent à leur tour des principes de la révolution américaine, et sont partisans d'une réforme parlementaire en Angleterre - «Baptistes, quakers, égalitaires et cosmopolites» ${ }^{1}$, qui inspirent Cartwright, Price, Paine ou Priestley, et Godwin. La question est donc de savoir quelle greffe helvétienne peut prendre 
sur le représentant, en 1793, d'une telle tradition britannique protestante, et quelles sont les raisons de sa réussite ou de son éventuel rejet.

Cette question a déjà donné lieu à des positions opposées qu'on rappelle ici brièvement. E. Halévy emploie à plusieurs reprises l'expression «disciple d'Helvétius» 2 à propos de Godwin, dont il fait le maillon reliant Helvétius à Owen dans l'histoire de la réception d'Helvétius en Angleterre. Certes, dans son interprétation de la philosophie de Godwin, Halévy ne fait pas d'Helvétius la seule inspiration de Godwin. Il note également que le système moral de Godwin s'oppose en dernière analyse à celui d'Helvétius, parce qu'il est régi par l'idée fondamentale de l'émancipation intellectuelle, tandis que le système d'Helvétius, comme celui de Bentham, un autre de ses «disciples» selon Halévy, l'est par la mécanique des passions égoïstes. Néanmoins Halévy maintient qu'il y a une relation de maître à disciple entre les deux auteurs.

D'autres commentateurs choisissent plutôt de développer les termes d'une opposition entre les deux philosophes. Un éditeur de Godwin, F. E. L. Priestley ${ }^{3}$, tente ainsi de démontrer méthodiquement que la métaphysique, la morale et la politique de Godwin se distinguent nettement d'une «école française» dont Helvétius serait la figure tutélaire, avec Rousseau et d'Holbach. Si on suit cette seconde interprétation, on doit insister fortement sur l'évolution de la pensée de Godwin. En effet, en 1793, la première édition de Political Justice s'ouvre explicitement sous les auspices de cette «école française», tandis que les éditions de 1796 et 1798 corrigent les chapitres qui, dans la première édition, allaient dans le sens de l'inspiration française. Godwin lui-même s'en explique dans la préface de la première édition, dans laquelle il admet que, pressé par une publication mise en route avant même que l'œuvre ne soit achevée, l'auteur (c'est-à-dire lui-même),

understood the proposition more completely as he proceeded, and saw more distinctly into the nature of the remedy. [...] This circumstance has led him into some inaccuracies of language and reasoning, particularly in the earlier part of the work, respecting the properties and utility of government $(P J, \mathrm{t} .1, \mathrm{p} \text {. VIII })^{4}$.

Cependant, tout en prenant acte des «repentirs» de Godwin, il faut pourtant lui accorder ce qu'il revendique lui-même dans la préface à la deuxième édition, révisée : «the spirit and great outlines of the work $[\ldots]$ remain untouched $[\ldots] \gg(P J, \mathrm{t} .1, \mathrm{p} . \mathrm{xv})$. Il nous faut alors chercher ce qui, au delà des différences doctrinales entre les éditions, aussi grandes soient-elles, permet à Godwin de se revendiquer encore d'un même «esprit» d'inspiration française. Halévy, qui adopte cette démarche, identifie chez Godwin et Helvétius ce qu'il appelle un même «intellectualisme» :

Pour Helvétius et pour [Godwin], en effet, les différences individuelles, dans le genre humain, s'expliquent toutes, ou presque toutes [...], non, comme pour Montesquieu, par des causes physiologiques ou physiques, mais par des causes morales, ou sociales. De là, pour l'un comme pour l'autre, l'importance extrême du problème politique, qui se ramène à un problème pédagogique : à la différence de l'éducation proprement dite, qui cesse d'agir après l'enfance, du livre, qui n'atteint qu'une élite, les institutions politiques exercent leur action sur tous, pendant toute une vie (Halévy, La formation..., t. 2, p. 52).

La parenté qui unit les deux philosophes est forte en effet : ils construisent le problème du changement politique dans les mêmes termes. C'est ce que nous établirons dans un premier temps. «L'intellectualisme» dont parle Halévy consiste à donner à l'histoire de l'entendement humain une dimension et un enjeu essentiellement politiques. À nos yeux, il s'agit donc plutôt d'une politisation de la théorie de la connaissance que d'une intellectualisation de la politique. Le problème politique peut être formulé ainsi : une fois établies la nocivité essentielle de la monarchie et la fausseté de ses principes, et donc la nécessité de la réforme des institutions politiques tant en France en 1758 (date de parution de De l'Esprit) qu'en Angleterre en 1793, des institutions politiques vraies sont-elles 
possibles? Si les institutions existantes (institutions éducatives, culturelles - le livre - et politiques) sont insuffisantes, sont-elles fausses, et que seraient des institutions vraies? Une politique de la vérité est-elle possible? On verra que les remaniements des différentes éditions de Political Justice ne changent pas fondamentalement les termes de la problématique générale. En revanche, et c'est ce qu'on verra dans un deuxième point, sur les trois éléments de réponse donnés par Godwin en 1793 et évoqués par Halévy (le livre, l'éducation, et les institutions politiques ellesmêmes), la position de Godwin évolue considérablement, jusqu'à s'opposer à celle d'Helvétius, au point que l'on peut certes maintenir que les deux auteurs posent le problème politique en termes d'éducation, mais à condition de préciser que cette dernière notion, problématique, recouvre deux projets divergents, qui révèlent des appartenances à deux traditions et à des références philosophiques distinctes. Helvétius propose ainsi une science de l'homme au service de la réforme des institutions, tandis que Godwin la lie au perfectionnement, ou à la perfectibilité des entendements individuels.

L'enjeu de cette divergence d'interprétation est le sens de la notion de perfectibilité, et l'histoire qu'on peut en faire. En effet, en distinguant plus fermement que ne le fait Halévy les positions finales d'Helvétius et de Godwin sur la notion d'éducation, sans se contenter de remarquer que le problème politique est compris à partir du modèle éducatif, on peut faire apparaître ce qui est apparu à Godwin comme les conditions de possibilité (ici théoriques seulement) de l'idée de perfectibilité de l'entendement, et les raisons de son usage parcimonieux chez Helvétius. Ainsi, le rapprochement opéré par Halévy sur la notion de perfectibilité indéfinie entre Political Justice de Godwin et l'Esquisse d'un tableau historique des progrès de l'esprit humain de Condorcet ${ }^{5}$, masque que l'idée sert deux projets politiques différents. L'extrême différence des projets éducatifs de ces deux philosophes ne peut être comprise que de ce point de vue.

\section{Les deux erreurs de Montesquieu, ou la construction d'un problème politique}

Dans son journal intime, Godwin décrit en 1791 le projet de Political Justice : «My original conceptions proceeded on a feeling of the imperfections and errors of Montesquieu, and a desire of supplying a less faulty work» 6 . Aux yeux de Godwin, dans Political Justice, les deux «erreurs» de Montesquieu ont été de considérer comme acquise la supériorité de la constitution monarchique anglaise, et d'attribuer des causes physiques aux différences entre les gouvernements, par conséquent entre les peuples. Quoi qu'il en soit de la justesse de cette interprétation de Montesquieu, elle révèle que Godwin fait de la lecture de Montesquieu le point de départ de son propre engagement dans la théorie politique. En d'autres termes, si Godwin refuse les «conclusions» de Montesquieu, ou ce qu'il présente comme tel, c'est cependant après Montesquieu que la nécessité d'une autre théorie politique se fait pressante. De quoi s'agit-il?

Le projet de Montesquieu dans l'Esprit des Lois est de faire apparaître l'ensemble des rapports que la loi exprime, pour rendre raison de cette dernière. De l'examen empirique des gouvernements, classés selon une typologie tripartite (républiques aristocratiques ou démocratiques; monarchies; despotismes), il ressort qu'une rationalité est à l'œuvre dans les lois positives dans chaque type de gouvernement, qui permet sa perpétuation. Par ailleurs, les républiques et les monarchies, si elles sont modérées 7 , peuvent être dites des gouvernements libres, c'est-à-dire qui garantissent un sentiment fondé de sécurité. Par conséquent, deux points sont acquis pour Montesquieu, que Godwin a parfaitement identifiés :

— l'inanité de la question du meilleur gouvernement d'un point de vue général, quand bien même Montesquieu a pu par ailleurs estimer, selon Godwin, que la constitution britannique est en 1748 le régime qui se rapproche le plus, parmi les gouvernements existant, d'un gouvernement modéré;

— l'importance du gouvernement, dont le «principe», c'est-à-dire la passion qui le meut, 
meut en même temps les sujets ou citoyens pris individuellement. Ainsi les sujets d'une monarchie durable et stable sont-ils tous régis par la passion de l'honneur, ceux d'une république par la vertu politique.

Godwin est si bien persuadé de cette dernière vérité philosophique qu'il ressent le besoin de la divulguer, par l'intermédiaire de l'écriture romanesque, à ceux qui ne sont pas susceptibles de lire des traités philosophiques. Things as They Are, or The Adventures of Caleb Williams, le roman de Godwin qui paraît immédiatement après Political Justice, doit répandre cette vérité «bien connue des philosophes» : «The spirit and character of government intrudes itself into every rank of society» $^{8}$. Le roman de Godwin montre, en situation, ou à la manière expérimentale, comment tous les sujets d'une monarchie obéissent à la passion de l'honneur, quelle que soit leur position sociale. Cependant, Godwin conteste que le régime monarchique et le principe de l'honneur puissent jamais assurer la liberté. C'est le sens philosophique et polémique du roman, dans lequel tous les personnages se trouvent dans des impasses morales ou affectives ${ }^{9}$. L'honneur meut certes les sujets des monarchies,

mais il ne

peut jamais créer des relations sociales harmonieuses. Il faut donc corriger Montesquieu, et poser à nouveau la question du meilleur gouvernement d'un point de vue général, puisque la monarchie, ou gouvernement de l'honneur, ne peut jamais être un régime modéré; quitte, on le verra, à conclure à l'incompatibilité de la liberté individuelle réelle et du gouvernement.

Or c'est précisément la conviction de la nocivité essentielle de la monarchie qu'en 1793 Godwin affirme avoir puisée dans la lecture des philosophes français, entre autres :

It is now twelve years since he [scil. Godwin] became satisfied, that monarchy was a species of government essentially corrupt. He owed this conviction to the political writings of Swift and to a perusal of the Latin historians. Nearly at the same time he derived much additional stimulus from several French productions on the nature of man [...] the Système de la nature, the works of Rousseau, and those of Helvétius (PJ, préface de 1793, t. 1, p. IX).

Quelques années auparavant, il notait dans son journal intime la satisfaction avec laquelle il lit «Helvétius, Rousseau, and others, the most popular authors in France», qui lui semblent supérieurs aux écrivains anglais et le font devenir «républicain» 10 . Avec Montesquieu, Godwin apprend que l'on peut connaître empiriquement, par l'observation des choses, les lois auxquelles elles obéissent; qu'il y a un système monarchique des choses, des lois et des hommes. Mais Rousseau et Helvétius lui montrent, selon lui contre Montesquieu, que non seulement on peut tirer de l'observation des «choses comme elles sont» la connaissance des lois qui les ordonnent, mais aussi un savoir «sur la nature de l'homme» («on the nature of man») qui montre la fausseté ou la nocivité de ce système monarchique. Il faut donc en passer par une théorie de la nature de l'homme pour découvrir une norme politique, à partir de l'observation des «choses comme elles sont», et du constat qu'elles sont au plus mal.

L'analyse de la faillite du régime monarchique est ce qui fait l'intérêt de l'œuvre de Godwin aux yeux de ses contemporains et ce qui fait de lui, à ses propres yeux, un continuateur de Rousseau et Helvétius. C'est pourquoi il peut maintenir en 1796, dans la seconde édition, que pour l'essentiel, «the spirit and great outlines of the work [. . . remain untouched [. . ] » $(P J, \mathrm{t} .1, \mathrm{p} . \mathrm{xv})$. Certes, par différence avec le monisme d'Helvétius, le dualisme est désormais affirmé fortement : il y a un système matériel et un système spirituel. Certes encore, le gouvernement, et toute institution, $\mathrm{y}$ compris l'institution éducative, sont présentés à partir de 1796 comme un obstacle au perfectionnement de l'homme. Or Godwin sait bien que cette dernière position fait désormais de lui 
un adversaire radical d'Helvétius; il l'indiquait dès ses dernières livraisons pour la première édition. Mais l'essentiel aux yeux de Godwin et de ses premiers lecteurs ${ }^{11}$ demeure : la

critique de l'ordre politique existant (la monarchie), acquis qui est généralement perçu en 1793 comme une importation française et une critique de Montesquieu, tant pour le contenu que pour la méthode.

Quant au contenu, il est exact qu'on chercherait en vain une critique principielle de la monarchie sous la plume de Montesquieu. Quant à la méthode, elle est, pour Godwin, indissociablement empirique et critique, ou normative. De la description des choses et de l'homme comme ils sont résulte l'énoncé d'une norme. C'est une démarche commune à Godwin et à ses prédécesseurs français. Leurs adversaires ne s'y sont pas trompés, et on retrouve sous leur plume, française ou anglaise, la même critique de cette démarche clairement identifiée. Côté français, on peut lire dans la comédie satirique Les philosophes une mise en scène de cette critique. Palissot y ridiculise le personnage de l'apprentie philosophe Cydalise, auteure d'un «livre de génie», «encyclopédie» morale dont le chapitre le plus «profond» s'intitule Les devoirs tels qu'ils sont et traite

En abrégé de l'esprit, du bon sens,/ Des passions, des lois et des gouvernements;/ De la vertu, des mœurs, du climat, des usages,/ Des peuples policés et des peuples sauvages;/ Du désordre apparent, de l'ordre universel,/ Du bonheur idéal et du bonheur réel (C. Palissot de Montenoy, Les Philosophes, Paris, Duchesne, 1760, p. 26).

Le titre (Les devoirs tels qu'ils sont) indique ironiquement la confusion qui serait faite par les philosophes entre le devoir-être et l'être. Côté anglais, l'Anti-Jacobin résume ainsi la doctrine de Godwin en 1798 : «Whatever IS, is WRONG + absolute and eternal perfectibility of man». Nous aurons l'occasion de voir que la formule est plutôt perspicace. Dans les deux cas, l'enquête empirique («tels qu'ils sont», ou «whatever IS») et la formulation d'une norme («les devoirs», ou «is WRONG») sont indissociablement accolés, ce qui est présenté comme un hybride paradoxal et risible. Il nous faut comprendre ce qui rend possible cette double démarche, présentée par Godwin comme une réponse à la deuxième erreur de Montesquieu (celle qui consiste à promouvoir un déterminisme matériel plutôt que circonstanciel ou moral dans l'explication des différences entre les peuples). La possibilité de construire une norme politique universellement valable par une démarche expérimentale (c'est-à-dire ici, fondée sur l'observation) est ainsi l'équivalent méthodique de la thèse affirmant le déterminisme moral. Si on peut en effet observer les malheurs causés par la morale de l'honneur, et que le principe de l'honneur est consubstantiel au système monarchique, il est possible d'en conclure la nocivité de la monarchie elle-même du point de vue du bonheur des particuliers; en outre, on peut supposer la réalité du déterminisme moral, ou l'influence des circonstances politiques pour ce bonheur. Les faits font ainsi droit,

non en un sens conservateur, mais parce que le fait du malheur indique qu'une prescription naturelle est bafouée; il pointe vers le droit.

Trois constats communs aux réformateurs français et anglais que nous avons évoqués dessinent les limites d'un espace théorique autorisant différentes réponses normatives :

1) la nécessité d'une évolution politique permanente, eu égard aux vicissitudes des choses mêmes; 
2) le rôle déterminant du gouvernement dans la possibilité pour les hommes de jouir en paix et de se comporter d'une façon vertueuse;

3) la réalité d'un déterminisme moral grâce auquel une science de la nature humaine est possible, et avec elle un infléchissement de l'histoire politique humaine vers plus de bonheur, par la réforme ou l'abolition

des

gouvernements.

À propos du premier constat, Montesquieu, là encore, formule un fait, ou un rapport, dont ses successeurs tireront une prescription normative. La vision dynamique qu'il donne des gouvernements, toujours près de tomber dans le despotisme ou la décrépitude, exprime la nécessité d'une vigilance, d'un ajustement perpétuels pour maintenir un éventuel état harmonieux. En politique comme en physique ${ }^{12}$, il n'y a pas de repos absolu, mais un rééquilibrage permanent du rapport des forces à l'œuvre dans le corps politique. L'aspiration à l'immobilisme est une illusion qui dissimule que tout équilibre est le produit de deux efforts continus et contrebalancés. Godwin affirme que dans la pensée également, il n'y a pas de repos, mais des avancées ou des reculs ${ }^{13}$. Dans les Lumières françaises, l'affirmation d'une régression politique s'appuie sur le prétendu constat empirique du dépeuplement et de la pauvreté grandissante, qui indique avec certitude qu'il y a une contradiction entre les conditions empiriques et les lois, et donc qu'un changement est nécessaire pour sauver l'État. Pour Helvétius,

en certains pays où les esprits, énervés par la superstition, sont paresseux et peu capables de grandes entreprises, on croit couvrir un homme du plus grand ridicule lorsqu'on dit de lui : c'est un homme qui veut réformer l'État. Ridicule que la pauvreté, le dépeuplement de ces pays, et par conséquent la nécessité d'une réforme, fait, aux yeux des étrangers, retomber sur le moqueur (De l'Esprit, II, 3, p. 66) ${ }^{14}$.

Dans ce passage, la clairvoyance du regard des étrangers sert à appuyer l'argument du dépeuplement comme signe de déclin ${ }^{15}$, conformément à une tradition littéraire à laquelle Montesquieu a donné ses lettres de noblesse

au XVIII ${ }^{\mathrm{e}}$ siècle. Helvétius poursuit en précisant que la «superstition» dont il est question ici, qui empêche la réforme, est la «stupide vénération des peuples pour les lois et les usages anciens» $(D E$, 11, 17, p. 158). En vérité, les lois ne sont pas sacrées, mais elles doivent pouvoir être revues; pour la plupart d'entre elles, elles ne sont même, de droit, que provisoires ${ }^{16}$. Godwin fait pareillement état d'une querelle fondamentale entre partisans du changement et partisans du conservatisme :

The question now afloat in the world respecting things as they are, is the most interesting that can be represented to the human mind. While one party pleads for reformation and change, the other extols in the warmest terms the existing constitution of society (Things as They Are, préface à la $2^{\mathrm{e}}$ édition, vol. 1, p. v).

Godwin prend parti pour les premiers, et exprime sa prise de parti tant à la manière expérimentale, par les situations mises en scène dans Caleb Williams, qu'à la manière théorique : «The wise man is satisfied with nothing. It is scarcely possible there should be an institution, in which impartial disquisition will not find defects» (PJ, t. 1, p. 266). 
Le réajustement permanent des institutions est rendu nécessaire par les vicissitudes continues avérées des temps et des circonstances, au rang desquelles il faut compter les révolutions américaines et françaises :

It would be strange if something of this kind were not requisite in the science of politics, after the concussion that the minds of men have suffered on this subject, and the materials that have been furnished, by the recent experiments of America and France (PJ, préface de 1793, t. 1, p. v).

Les matériaux nouveaux offerts par les révolutions font progresser la science politique, et rendent nécessaire une perpétuelle réforme, au sens d'un ajustement des artifices politiques à l'état de la science.

Selon le second constat, de cette mutabilité politique permanente et nécessaire, il ressort que le «gouvernement» (institutions publiques et exercice du pouvoir) joue un rôle crucial dans le bonheur des individus. Dans la version de 1796 du premier chapitre de Political Justice, Godwin affirme d'abord qu'il doit cette thèse aux «plus grands patriotes» et aux «écrivains politiques les plus célèbres», c'est-à-dire dans un premier temps Locke, Sydney et Paine : ces derniers prônent la légitimité du gouvernement, mais refusent la thèse de son efficace positive dans la poursuite individuelle du bonheur. Puis Godwin précise que les défenseurs «par excellence» de la thèse de l'influence du gouvernement sont Rousseau et Helvétius, qui s'en remettent à un gouvernement sage pour l'exercice individuel de la moralité et la jouissance des plaisirs :

they have treated morality and personal happiness as a science, and politics as a different one. But, while they have considered the virtues and pleasures of mankind as essentially independent of civil policy, they have justly remarked, that the security with which the one can be exercised and the other enjoyed, will be decided by the wisdom of our public institutions and the equity with which they are administered (PJ, t. 1, p. 3-4).

Dans un premier temps, le début du résumé de Godwin surprend : il est difficile de comprendre comment Godwin, qui a lu De l'Esprit et De l'Homme, peut croire que Rousseau et Helvétius séparent la politique et la morale. Godwin ne peut pas ignorer que pour Helvétius «la morale n'est qu'une science frivole, si l'on ne la confond avec la politique et la législation» (DE, II, 15, p. 152, nous soulignons). De même, en lecteur d'Émile qu'il est peut-il oublier que pour Rousseau il «faut étudier la société par les hommes, et les hommes par la société : ceux qui voudront traiter séparément la politique et la morale n'entendront jamais rien à aucune des deux» (IV, p. 523$)^{17}$ ? Pour comprendre l'interprétation que Godwin donne des deux philosophes français, il faut porter son attention sur la fin de la citation. Helvétius et Rousseau pensent des conditions de possibilité politiques à l'exercice de la vertu (ou de la justice) et à la jouissance des plaisirs, mais ni la nature de la vertu ni celle des plaisirs n'est déterminée par la politique, au sens ou elles ne sont pas déterminées par les lois. Or pour Godwin, la dimension artificielle de la jonction entre les vertus et les plaisirs d'un côté, et la politique de l'autre, ne peut que présupposer une séparation entre science politique et science morale. Dans le cas d'Helvétius, il est exact que le gouvernement (c'est-à-dire «l'assemblage des lois», $D H, \mathrm{IX}, 9, \mathrm{p} .785$ ) forme un édifice artificiel, ou conventionnel, qui vise à assurer le service de l'intérêt du public par le mécanisme de l'intérêt personnel. Les individus, mus par la seule recherche de leur intérêt personnel, ou de leur plaisir, ne sauraient en effet jamais servir l'intérêt public s'ils n'y voient pas le moyen de mieux assurer la jouissance de leurs plaisirs et la fuite de ce qui leur est pénible. En un sens, on peut dire que la politique reste extérieure à la morale, car le service de l'intérêt public pour lui-même n'est jamais un motif de l'action des particuliers. Ce n'est pas un motif à prendre en compte pour une science morale des lois de l'action des particuliers. Cependant si les particuliers ont intérêt à la justice, c'est-à-dire au respect des conventions fondant la propriété privée, le triomphe de la justice ne peut être assuré que par le moyen de la politique et 
d'institutions puissantes. La science morale n'est donc rien si elle n'est pas réalisée dans un jeu du pouvoir. Godwin a donc raison de donner à nouveau l'interprétation suivante de la pensée d'Helvétius en 1796, dans le deuxième livre de Political Justice :

The nature of happiness and misery, pleasure and pain, is independent of political institution. It is immutably true, that whatever tends to procure a balance of the former is to be desired, and whatever tends to procure a balance of the latter is to be rejected $(P J, \mathrm{t} .1, \mathrm{p} .171)$.

Dans les premiers chapitres, il s'inscrit dans la lignée helvétienne en faisant apparaître le rôle du gouvernement : de lui, de la sagesse des législateurs qui auront ou non établi un «plan of power» empêchant la corruption et la tyrannie (PJ, t. 1, p. 3-4), dépend le bonheur des citoyens, la jouissance des plaisirs et l'exercice de la vertu. En raison de la place qu'ils lui accordent tous deux, Godwin n'a pas tort non plus d'affirmer qu'il emprunte à Helvétius, qui l'aurait lui-même empruntée à Rousseau, l'idée selon laquelle «the imperfections of government [are] the only perennial source of the vices of mankind» $(P J$, t. 2, p. 129). Néanmoins Helvétius accorderait que le gouvernement et les lois sont la source des vices de l'homme moderne, qu'il appellerait par conséquent plutôt sa corruption politique, mais pour ajouter qu'il pourrait être aussi la source de sa vertu, ou capacité à servir l'intérêt public. Avec exactitude, Godwin gratifie immédiatement Rousseau d'être le seul à avoir vu que le gouvernement, «however reformed», ne saurait exercer d'action bénéfique. Il affirme ainsi son opposition à la conséquence tirée par Helvétius de l'importance que tous attribuent au gouvernement.

Enfin, en ce qui concerne le troisième constat, quoi qu'il en soit de l'évaluation du rôle du gouvernement (qu'il exerce une influence nécessairement néfaste ou qu'il puisse être l'instrument principal pour le bonheur des hommes), affirmer la réalité de son influence, c'est accepter la thèse d'un déterminisme moral. Les institutions humaines exercent une action en retour sur les hommes. Godwin maintient dans la deuxième édition ce principe tiré de l'examen des «faits historiques» : «The characters of men originate in their external circumstances» (PJ, t. 1, p. 24-51). Mais si l'on veut mesurer exactement l'influence des institutions politiques parmi ces «circonstances extérieures», il faut passer par une théorie complète de l'entendement humain («human mind»). Dans un premier temps, Godwin se libère de la question substantialiste qui n'importe pas, pour mieux faire apparaître l'enjeu politique de toute théorie de la connaissance.

Répétant le geste théorique effectué par Helvétius, Godwin se place sous l'autorité de Locke, Hume et Berkeley pour s'autoriser le terme «mind»sans prendre parti sur la nature matérielle ou spirituelle de la série des événements (qu'on les appelle pensées, idées, perceptions ou sensations) qui forment l'entendement. Godwin concède que nous sommes dans l'incertitude à propos des causes de nos sensations, et de leur éventuelle similitude substantielle avec les idées qu'elles produisent : «We know nothing of the substance or substratum of matter [. . ]». Cependant, on peut se dispenser de prendre position sur cette question métaphysique,

et probablement oiseuse, et parler d'un entendement humain réglé par des lois propres :

our thoughts, ideas, perceptions, or sensations (by whatever term we choose to express them) [...] are ordinarily linked together, so as to produce the complex notion of unity or personal identity. Now it is this series of thought linked together, without considering whether they reside in any or what substratum, that is most aptly expressed by the term mind (PJ, p. 25-26). 
La proximité avec les définitions de la matière et de l'esprit données par Helvétius, ainsi que l'indifférence proclamée pour la question de la détermination substantielle est frappante, mais elle est probablement à rapporter aux références anglaises communes ${ }^{18}$.

Le point important ici est la subordination de l'histoire de l'entendement humain à la problématique politique. Ce n'est que dans la perspective d'une éventuelle action politique réformatrice que Godwin consacre un chapitre à l'entendement humain. La théorie de la connaissance l'intéresse pour autant qu'elle doit permettre de mesurer si une action politique institutionnelle est possible et souhaitable pour le bonheur des hommes. Il importe de savoir à quelles conditions les hommes pensent et connaissent la vérité dans la seule perspective d'assurer à tous ces conditions d'exercice de la pensée juste, car tous doivent pouvoir devenir ce qu'ils sont toujours déjà, mais non toujours pleinement : des êtres pensants. Or c'est précisément cette inscription de l'esprit humain dans une histoire politique, à des fins réformatrices, qui constituait déjà l'originalité philosophique d'Helvétius, et le distinguait de Rousseau aux yeux de Godwin. En effet, si Rousseau est gratifié d'être le seul a avoir aperçu la nocivité de tout gouvernement dans ses Discours, son génie pourtant «le quitte» dans ses ouvrages «expressément politiques ( $\mathrm{Du}$ Contrat social et Considérations sur la Pologne)» ${ }^{19}$, qui développent la politique sans la théorie de la connaissance, tandis que De l'Esprit place la théorie de l'esprit sous les auspices du questionnement politique. Pour Godwin de la même manière, et ce même à travers les évolutions de sa philosophie, il s'agit toujours, par les moyens de l'histoire, de la philosophie ou de la littérature, de connaître l'homme pour savoir ce dont est capable l'homme social ${ }^{20}$. La question de la réforme requiert la fondation d'une science nouvelle, indissociablement morale et politique, qui doit dire ce qu'il est souhaitable d'instituer. En d'autres termes, des institutions scientifiques, au sens où elles sont dictées par la science de l'homme, sontelles possibles? Une politique de la vérité est-elle possible?

On voit à présent l'espace théorique à l'intérieur duquel Godwin va développer sa propre thèse, anti-helvétienne, de la nocivité essentielle de toute institution, qu'elle soit politique, religieuse, ou éducative. Il utilise les philosophes français, en particulier Helvétius et Rousseau, pour cau-

tionner les prémisses de sa philosophie : nocivité de la monarchie, vicissitude essentielle du politique, influence cruciale du gouvernement dans le bonheur des hommes. Le constat de la nocivité du système monarchique démontre l'influence des gouvernements sur le bonheur des individus et indique que si certains régimes sont contradictoires avec la nature même de l'homme, d'autres lui sont peut-être favorables. L'éducation des enfants offre le modèle pour penser l'efficace du gouvernement dans la vie des particuliers. Puisque les hommes sont visiblement éducables, ne peut-on pas dire que l'effet des gouvernements sur la vie des particuliers est un type d'éducation? Ou plutôt, ne peut-on pas penser tout déterminisme moral comme un processus éducatif en un sens large, et l'éducation de l'enfance comme une forme temporaire, limitée de l'éducation? Le gouvernement apparaît alors comme le grand éducateur, non parce qu'il est instructeur et investi d'une mission éducative au sens strict, mais parce qu'il est législateur et que les lois instruisent sans cesse les particuliers sur leur intérêt. Mais alors les questions : qu'est-ce qu'apprendre? et surtout : qu'est-ce qu'une éducation libre? signifient : qu'est-ce qu'être gouverné? et : qu'estce que le régime de la liberté?

La réponse de Godwin dans Political Justice n'est pas un «patchwork de fragments de Locke, Helvétius, Rousseau, d'Holbach, Mably, Paine, Wallace, Beccaria, et Sir Thomas More», comme on a pu l'écrire au début du $\mathrm{XX}^{\mathrm{e}}$ siècle $^{21} \ldots$ Elle est originale, et révèle son appartenance à une tradition bien plutôt anglaise, issue de la dissidence religieuse, que française, issue des Lumières, $a$ fortiori des Lumières matérialistes. On pourrait dire avec Halévy que Godwin, comme Helvétius, 
ramène le problème politique au problème pédagogique, et pense l'influence des institutions politiques en termes éducatifs, mais il faut préciser que pour le premier l'éducation est un phénomène presque entièrement néfaste, dans la mesure où il repose la plupart du temps sur un rapport d'autorité autre que celui du seul savoir :

As long as parents and teachers in general fall under the established rule, it is clear that politics and modes of government will educate and infect us all. They poison our minds, before we can resist, or so much as suspect their malignity. [...] So false is the opinion that has too generally prevailed, that politics is an affair with which ordinary men have little concern $(P J, \mathrm{t} .1$, p. 49-50, nous soulignons).

\section{Réforme des institutions ou perfectionnement des entendements}

Contrairement à ce qu'on pourrait penser immédiatement, et contrairement à une idée reçue concernant les Lumières, poser le problème politique en termes éducatifs conduit à minimiser le rôle de l'éducation prise comme instruction et éducation de l'enfance. Nous allons voir comment l'alliance du paradigme éducatif et de la visée émancipatrice, ou éclairée,

de la politique, produit ce résultat paradoxal. Ce dernier prend en outre deux formes politiques distinctes chez Helvétius et chez Godwin.

Helvétius partage explicitement en deux parties la question du plan de législation $(D H, \mathrm{IX}, 1, \mathrm{p}$. 744), le «plan of power» évoqué plus tard par Godwin. La première partie enquête sur les lois propres à rendre les hommes le plus heureux possible. Sa méthode est «géométrique», c'est-à-dire qu'elle fait abstraction des caractéristiques historiques des sociétés; elle les simplifie pour obtenir les lois de la nature humaine et de son bonheur, à la manière du géomètre qui donne les lois du mouvement, abstraction faite de la résistance de l'air, de la densité du corps, etc. Helvétius pense avoir fourni cette partie géométrique de la science de la législation avec De l'Esprit. La vérité en question se ramène au principe unique de la sensibilité physique, en vertu d'un idéal de science condillacien dans lequel toutes les vérités peuvent se rapporter à un principe, ou fait, unique. Audelà de la variabilité essentielle de la majeure partie des lois, de la dimension conventionnelle de la justice, c'est-à-dire de la vertu, il y a une science vraie de la nature humaine, immuablement et éternellement vraie : l'homme, doté de la seule sensibilité physique, est le produit et le moteur de son histoire. La deuxième partie pose expressément la question de la réforme, ou comment «faire insensiblement passer un peuple de l'état de malheur qu'il éprouve à l'état de bonheur dont il peut jouir. [...] La solution doit être différente selon la forme différente de chaque État» (ibid.).

Le philosophe indique quelles sont les questions précises auxquelles il faut répondre pour établir les lois de transition, mais son travail concerne essentiellement la partie géométrique, celle qui, invariable, est susceptible d'atteindre la vérité. La première et la seconde partie de la science de la législation sont intimement liées, car «si la justice et la vérité sont sœurs, il n'est de lois réellement utiles que les lois fondées sur une connaissance profonde de la nature et des vrais intérêts de l'homme» (DH, VII, 2, p. 608). La richesse des sociétés, la force de la contrainte de «police» exercée sur ses membres, deux conditions essentielles pour qu'une société moderne soit vertueuse et heureuse, ne sont rien si les législateurs sont ignorants. «Les vertus fuient les lieux d'où la vérité est bannie» (DH, IX, 13, note, p. 801).

Cette exaltation de la vérité implique que la première de toutes les réformes à faire, quel que soit l'état empirique d'une société donnée, est l'établissement d'une liberté totale de pensée et de parole : «En morale [. . .] la seule chose réellement nuisible est la non-recherche [du vrai]. Qui prêche l'ignorance est un fripon qui veut faire des dupes» (DH, VII, 4, note a, p. 615). La vérité 
progressant toujours d'une manière pacifique $(\mathrm{DH}, \mathrm{X}, 8$, passim.), on est assuré que le progrès de la vérité produit toujours plus de bien que de maux, qu'il est un progrès de l'humanité en général. Une fois la liberté de pensée établie, les législateurs changeront petit à petit celles des lois qui sont variables en raison de l'imperfection de

notre science de la législation. Ce sont «les lois civiles et criminelles; celles qui regardent l'administration des finances, le partage des biens, les testaments, les mariages, etc.» Il ne restera que quelques lois variables par leur nature, qui devront être perpétuellement ajustées aux circonstances. Ce sont les lois qui concernent «le commerce, la discipline militaire, les impôts, etc.» (DH, X, 7, p. 909).

Un tel plan de législation contient nécessairement un plan d'éducation, mais ils ne peuvent se précéder ni l'un ni l'autre. Si l'on change l'éducation sans changer les lois, par exemple si l'on diffuse des catéchismes philosophiques qui font voir la vraie nature et les capacités des hommes, mais sans changer les lois d'une monarchie qui les classe et les récompense selon les faux critères de la naissance et du privilège, alors «l'éducation» au sens strict reçue dans l'enfance entrera en contradiction avec «l'éducation» au sens large que les hommes reçoivent, à partir de l'adolescence, des circonstances inchangées des mœurs et des lois ${ }^{22}$. Dans une telle contradiction, l'éducation du monde adulte l'emporte nécessairement, puisque les hommes cherchent à satisfaire leur intérêt, et sont plus assurés de trouver le plaisir et d'éviter la douleur en se conformant aux mœurs et lois actuelles qu'à des enseignements passés qui ne trouvent pas de répondant réel dans le présent. On n'aura alors rien fait progresser. À l'inverse, réformer les lois implique de se donner le système éducatif qui leur correspond. Si, comme Helvétius l'affirme, «l'éducation peut tout» $(\mathrm{DH}, \mathrm{X}, 1, \mathrm{p}$. 879), il ne peut s'agir que de l'éducation au sens large, celle qui est imposée par la totalité des circonstances, qui forme la «position» des individus ( $D H, \mathrm{X}, 1, \mathrm{p} .879-883)$. Parmi les circonstances qui font des hommes ce qu'ils sont, les hommes peuvent agir par les lois, lesquelles à leur tour ne doivent jamais précéder les lents progrès de la vérité, accomplis grâce à la liberté de pensée et à la réforme progressive des lois variables en lois invariables. Helvétius ramène le problème politique au problème éducatif, au prix d'une distinction entre l'éducation de l'enfance, au sens étroit, et l'éducation de l'âge adulte, qui inclut l'action de toutes les circonstances, au premier rang desquelles le gouvernement. L'éducation de l'enfance voit son importance minorée, car elle ne saurait jamais tenir lieu de détermination en dernière instance dans les motivations des actions individuelles. Cette détermination dernière est celle de l'intérêt personnel, auquel les particuliers obéissent sans errer. Il ne s'agit donc pas non plus d'apprendre aux hommes ce qu'ils savent déjà reconnaître et poursuivre leur intérêt personnel —, mais de leur donner les moyens politiques de faire ce qu'ils savent déjà faire sans se nuire réciproquement, par l'effet d'un système politique faussement construit qui les dresserait les uns contre les autres, ou contre leur propre nature.

La présentation que l'on vient de donner insiste singulièrement sur le rôle de la science et des progrès de la vérité. Suivant ce point de vue, on aperçoit bien la proximité des deux problématiques, ainsi que les raisons

de leur divergence finale. Comme point de départ, Godwin accorde que le gouvernement joue un rôle crucial dans le bonheur de l'homme : Caleb Williams et le premier livre de Political Justice démontrent que les institutions politiques actuelles sont la cause du mal moral. De là, il affirme que toute institution politique est toujours néfaste, en d'autres termes, qu'une réforme des institutions politiques peut certes les rendre moins nocives, mais jamais bonnes. L'horizon désigné pour le bonheur est celui d'une société sans gouvernement. 
Quelles sont les voies pour y parvenir, puisque ce ne sont pas, ou pas seulement, celles de la réforme? La meilleure indication se trouve dans l'index que Godwin a lui-même dressé pour Political Justice : à l'entrée Réforme politique, nous sommes renvoyés à une autre entrée : «Reform, political : see Improvements». La première voie vers le progrès semble n'être rien d'autre que le progrès rationnel individuel, assuré par la liberté de pensée et de parole. Pour autant, il ne s'agit pas bien entendu de ranger Godwin dans le camp des conservateurs, mais non plus dans celui des utopistes. Si les réformes institutionnelles ne suffisent pas, et bien que les institutions soient à compter au rang des obstacles à une véritable liberté du jugement individuel, ce sont pourtant de nombreuses réformes institutionnelles, signes d'un perfectionnement général, qui mènent peu à peu à l'abolition de toute institution politique. C'est ce qu'indique une sousentrée de «Improvements, political, mode in which they are to be realized» $(P J$, index, t. 1, p. 1 et p. xxxix), qui nous renvoie au chapitre 7 du troisième livre de Political Justice, «Of Forms of Government». Dans ce chapitre, Godwin oppose la réforme non à la conservation, mais à la révolution : la réforme permet aussi d'échapper aux excès de toute révolution, telle la Révolution française qui en septembre 1792 horrifie les réformateurs anglais. Le perfectionnement des entendements individuels mène en effet à de «nombreuses réformes»:

if conviction of the understanding is the compass which is to direct our proceedings in the general affairs, we shall have many reforms, but no revolutions. [...] Revolutions are the product of passions, not of sober and tranquil reason (PJ, t. 1, p. 244).

En dernière instance, Godwin n'est donc pas un philosophe de la réforme, mais du perfectionnement, pas un philosophe des institutions, mais de l'entendement. «What I should desire is not by violence to change its [=society] institutions, but by discussions to change its ideas» $(P J, \mathrm{t}$. 2, p. 539). Helvétius, qui a consacré un chapitre entier à établir que la vérité progresse lentement mais sans produire de troubles, souscrirait à ce refus de la violence, et partagerait la conviction qu'il faut que la vérité progresse par la libre discussion publique pour que progresse la société. Mais pour Helvétius, des institutions incarnant l'état d'avancement de la science, ou

des lumières, sont possibles, et in fine des institutions vraies. C'est que pour lui, le progrès a un terme, qu'il trouve dans la science vraie, qui s'incarnera dans des institutions vraies, tandis que pour Godwin, le perfectionnement est réellement infini.

Dans la deuxième édition de Political Justice, Godwin généralise sa position à toute institution, c'est-à-dire également à l'institution éducative. On emprunte ici à B. Binoche la définition de l'institution chez Godwin : «une institution, c'est un régime régulier de contraintes. Une institution positive, c'est un régime régulier de contraintes ordonné par la loi» ${ }^{23}$. Toute institution, par sa nature même, devient à un moment donné un obstacle dans l'histoire des progrès des entendements humains. Les trois causes du progrès évoquées par Halévy, qui sont autant d'institutions, «positives» ou non : la littérature, l'éducation et les institutions politiques, faisaient l'objet dans l'édition de 1793 d'un chapitre qui est entièrement supprimé dans l'édition de 1796. Le chapitre 4 du premier livre : «Three Principal Causes of Moral Improvement Considered: I. Literature II. Education III. Political Justice» disparaît et est remplacé en 1796 par le chapitre 5 du même livre : «The voluntary actions of men originate in their opinions». Le progrès moral général ne peut apparemment plus provenir que d'une action libre, «volontaire», qui trouve ses motifs dans des convictions rationnelles. Le nouveau chapitre est clos par un passage sur la perfectibilité de l'homme.

La correction touchant la littérature n'est pas un reniement, mais il y pointe une difficulté que Godwin tente de résoudre par sa propre pratique de l'écriture romanesque. Comment, en effet, la littérature, qui joue sur des processus d'identification, de manipulation des émotions, peut-elle 
devenir une littérature de la liberté? Si l'on peut dire que la littérature est une institution de la culture, est-il possible d'inventer des règles, c'està-dire des formes littéraires qui ne contraignent pas la liberté de jugement du lecteur? L'écriture subjective, à la première personne, de Caleb Williams et des romans qui le suivent, est une stratégie narrative pour conjurer la voix omnisciente et manipulatrice d'un narrateur. Mais la littérature n'est plus en elle-même, comme Godwin l'avait présentée en 1793, une cause de progrès. Elle peut offrir des matériaux à la pensée, mais dès qu'elle prétend penser à la place du lecteur, elle le corrompt. Toute position de surplomb par rapport au jugement individuel autonome empêche le progrès, et par là même non seulement empêche, mais corrompt. «Where I make the voluntary surrender of my understanding, and commit my conscience to another man's keeping, [...] I then become the most mischievous and pernicious of animals $[. .$.$] »$ $(P J$, t. 1, p. 232-233).

Ce droit absolu au jugement privé, dont on a beaucoup parlé chez Godwin, assimile toute position d'autorité à une relation de tutelle, et par là même la rend illégitime. Mais on voit bien qu'il met en difficulté et oblige à repenser à nouveaux frais la relation pédagogique au sens strict, que

(304)

Godwin avait dans un premier temps incluse dans les causes du progrès. Que peut être une éducation parfaitement libérale, donc progressiste?

L'éducation compte quant à elle au nombre des institutions qui imposent des contraintes aux entendements individuels, fussent-ils ceux des enfants, que Godwin ne distingue pas fondamentalement de ceux des adultes. La permanence de la thématique pédagogique dans toute l'œuvre et la vie de Godwin ne prend sa véritable dimension que si on la rapporte à la question politique dont elle dépend. En réalité, c'est la vie de tous les individus qui est placée sous le signe de cette difficulté pédagogique : si l'opinion selon laquelle la politique concerne peu les hommes ordinaires est fausse (puisque le gouvernement s'insinue dans tous les rangs de la société, comme on l'a vu plus haut), il est par conséquent tout aussi faux que la question de l'éducation ne les concerne pas non plus. Il ne s'agit en effet que d'une seule et même question. La reprise dans des œuvres ultérieures de la question pédagogique est ainsi une reprise d'un incessant avec Helvétius, sur la notion de l'éducation des circonstances et son corollaire, l'égalité des esprits. Ainsi Thoughts on Man, His Nature, Productions and Discoveries, publié par Godwin en 1831, discute-t-il à nouveau la thèse de l'égalité naturelle des esprits, et aux yeux d'un Anglais de cette époque (par exemple, Godwin, mais aussi James Mill ${ }^{24}$ ), cette thèse est celle du seul Helvétius. La thèse est déjà discutée et contestée dans The Enquirer ${ }^{25}, 34$ ans plus tôt. Ce qui importe alors pour Godwin est de montrer que l'on peut affirmer l'existence d'inégalités naturelles, ou plutôt d'inégalités au moment de la naissance, et maintenir en même temps, comme Helvétius, que tous les enfants sont également capables de développer une capacité critique de juger. La reprise de la thèse helvétienne de l'égalité des esprits, dans la première édition de 1793, apparaît alors comme un emprunt ponctuel qui n'était destiné qu'à appuyer la thèse principale de la perfectibilité de tous les hommes. Il faut mettre en œuvre une pédagogie dans laquelle la relation d'autorité entre le précepteur et l'élève est fondée sur la seule autorité de la raison elle-même. L'enfant ne soumet son jugement qu'à un jugement non pas plus développé, plus mûr, plus accompli, mais à un jugement plus informé : «The deference of a child becomes vicious, whenever he has reason to doubt that the parent possesses essential information, of which he is deprived» $(P J$, t. 2, p. 236).

Pour Godwin, un tel projet ne peut pas, par définition, être mené dans le cadre d'une éducation publique nationale. On comprend à présent pourquoi Godwin rend hommage au Rousseau de l'Émile mais se désole de l'absence de génie des Considérations détachées sur le gouvernement de Pologne, qui développent l'idée d'une éducation publique, certes «négative», mais dont les responsables sont nommés et révoqués par un collège de magistrats (chapitre 4). Godwin argumente 
longuement son opposition à toute éducation nationale publique $(P J, \text { t. } 2 \text {, p. 296-304 })^{26}$ : avant tout,

l'éducation est la manière par laquelle les gouvernements ont pris l'habitude d'interférer et d'influencer les opinions. En tant que membre d'une famille de «Dissenters», Godwin a pu faire l'expérience directe d'une telle contrainte des consciences, dans les opinions religieuses en particulier. L'alliance du gouvernement et de l'éducation est «an alliance of a more formidable nature, than the old and much contested alliance of church and state» (PJ, t. 2, p. 302). Elle ne peut que servir les vues du gouvernement, qui cherche nécessairement à se perpétuer, et à sacraliser sa Constitution. Or les élèves «should be led to venerate the truth, and the constitution only so far as it corresponds with their uninfluenced deductions of truth» (ibid.).

Ensuite, s'il est certain que dans un premier temps la diffusion des savoirs aura des effets bénéfiques, l'utilité de ces savoirs et donc de l'institution scolaire elle-même diminuera à mesure même qu'ils seront répandus. Plus encore, un enseignement public institutionnalisé tend nécessairement à figer les esprits dans les erreurs du passé : Godwin en veut pour preuve l'état de l'enseignement dans les Universités, qui serait un reflet fidèle de l'état du savoir cent ans auparavant... L'éducation publique n'a jamais enseigné à ses élèves à soumettre toute proposition à l'épreuve de l'examen critique, mais à retenir des vérités. Or aucune vérité n'est jamais assez sûre pour être légitimement inculquée. Les sciences elles-mêmes ne sont pas un état indépassable du savoir, par conséquent les sciences morales et politiques non plus. Tout apprentissage dogmatique, fût-il scientifique, se ramène à l'apprentissage d'un «catéchisme» : «refer them to reading, to conversation, to meditation; but teach them neither creeds nor catechisms, either moral or political».

Dans ce contexte, on comprend que la place réduite que Godwin réserve à l'éducation révèle une pensée qui discrédite toute volonté d'améliorer l'homme et son bonheur par des voies indirectes telles que les lois ou l'institution éducative. Le seul progrès réel est accompli par l'individu luimême. Le reste n'est que «perpetual pupillage». La théorie de l'éducation, et donc avec elle toute théorie de la réforme des institutions politiques, repose sur l'idée que «unpatronised truth is inadequate to the purpose of enlightening mankind». Les Lumières, dans leur version godwinienne, ne décrivent pas nécessairement un processus éducatif conçu comme «l'éclaircissement» des entendements populaires ou mineurs, mais plutôt l'appropriation continuelle, par un entendement toujours déjà majeur, en possession de ses moyens, de la vérité.

Une distinction fondamentale apparaît donc, par l'intermédiaire de la question éducative, sur la question même des Lumières et de leur diffusion. Helvétius et Godwin reprennent sans cesse la même question, qui envisage d'un même point de vue pouvoir politique, histoire de l'entendement humain, et science morale du bonheur de l'homme. Mais la notion de perfectibilité indéfinie est doublement secondaire chez Helvétius. Dans un premier temps, l'idée même de perfectibilité (et donc le terme lui-

même) n'est pas nécessaire au bonheur de l'homme civil, puisque celui-ci n'a pas besoin de devenir meilleur pour être plus heureux. Il lui suffit de se trouver dans un dispositif politique construit conformément aux réquisits de sa nature, lesquels ont été mis au jour par la science morale et politique achevée. La raison peut produire de tels dispositifs artificiels plus ou moins aptes à produire le bonheur des individus; elle ne saurait certes jamais les imposer par la force contre les mœurs, mais il n'est pas nécessaire que les citoyens soient partie prenante d'un tel dispositif en connaissance de cause. C'est donc la science qui est perfectible, et Helvétius utilise plus aisément l'expression perfection ou perfectionnement «des sciences», que «des entendements individuels». 
Les savants font progresser la science, les philosophes en offrent les éléments qui, constitués en un dictionnaire, offrent à tous les éléments d'un exercice plus sûr du jugement, mais essentiellement identique à l'exercice commun du jugement. En outre, la science elle-même n'est pas soumise à un perfectionnement indéfini. Elle peut atteindre un point de perfection - ce qui fonde la différence entre l'apprentissage d'un catéchisme et la répétition de préjugés.

Un tel point fixe est impossible pour Godwin. Que l'homme soit essentiellement perfectible ne signifie pas que la perfection soit jamais accessible à l'homme, au contraire :

By perfectible, it is not meant that he is capable of being brought to perfection. But the word seems sufficiently adapted to express the faculty of being continually made better and receiving perpetual improvement. [ . . ] If we could arrive at perfection, there would be an end to our improvement (PJ, t. 1, p. 93).

Dans ce processus de perfectionnement continu, il n'y a même quasiment pas d'enfance. L'homme de Godwin doit vivre une sorte de majorité perpétuelle : l'enfant lui-même n'est pas soumis à une forme adulte de la raison, qui impliquerait que la sienne soit mineure, mais seulement à une raison plus informée; ce qui signifie d'un point de vue politique que les moins avancés sur le chemin du jugement autonome sont toujours assez avancés pour refuser que l'on pense à leur place — à moins d'une véritable différence d'information.

On peut se demander si cette opposition ne relève pas, en dernière analyse, d'une différence entre un courant des Lumières françaises, réformatrices et étatistes, et un courant des Lumières anglaises, aux sources religieuses dissidentes ou réformées, porteuses de l'idée de perfectibilité, dont les accointances sont plutôt de l'autre côté de l'Atlantique. En fait, il y a plusieurs manières d'entendre cette même notion, et les positions des uns et des autres sur l'éducation, en particulier l'éducation publique, en sont une conséquence et, pour nous, un indice. Lorsque Condorcet annonce, dans la neuvième époque de l'Esquisse, le développement de la «doctrine de la perfectibilité indéfinie de l'espèce humaine», il se réfère à Turgot,

mais aussi à Price et Priestley ${ }^{27}$, identifiant la racine anglaise de la doctrine. Il ne cite pas Godwin qu'il n'a certainement pas pu lire dans le court laps de temps qui sépare la publication anglaise et son décret d'arrestation. En revanche, Godwin fait référence à Condorcet dans la troisième édition de Political Justice, pour noter une différence entre lui-même d'un côté, et Bacon et Condorcet de l'autre : ces deux derniers auteurs ont d'après lui fondé leur espoir dans la possibilité de reculer le terme de la vie «rather upon the growing perfection of art, than, as is here done, upon the immediate and unavoidable operation of an improved intellect» (PJ, t. 2, p. 250, note). C'est dire que pour Godwin, la perfectibilité est à rapporter aux entendements eux-mêmes, tandis que Bacon et Condorcet la rapportent à l'état des techniques et des sciences. On retrouve donc ici, sous la bannière commune de la perfectibilité, la même opposition que celle qui, sous la bannière commune de la critique anti-monarchiste, distinguait Helvétius d'un côté, partisan d'un artifice, d'une technique politique pour le bonheur des citoyens, et Godwin de l'autre, qui prône, à l'horizon de la perfectibilité indéfinie, un auto-gouvernement des individus. Plus tard encore, Cabanis reprendra le flambeau helvétien, en défendant une institution de la vérité par un système éducatif censé refléter un système vrai des sciences. Mais il se distingue alors de Condorcet, pour qui les distinctions disciplinaires et institutionnelles sont destinées à disparaitre, à l'horizon d'une perfectibilité indéfinie $^{28}$. Les véritables héritiers d'Helvétius ne sont décidément pas des penseurs du progrès infini, mais bien d'une technique politique à prétention scientifique.

\section{Notes}


1 Elie Halévy, La formation du radicalisme philosophique [1901-1904], 3 tomes, édition révisée dirigée par Monique Canto-Sperber, Paris, Presses Universitaires de France, 1995. Ici t. 1, ch. 3, 2.

2 Halévy, La formation, t. $1, \quad$ p. $\quad 30, \quad$ ou $\quad$ t. $\quad 2, \quad$ p. $\quad 90$.

3 William Godwin, Enquiry Concerning Political Justice and Its Influence on Morals and Happiness, facsimile of the 3rd edition [1798], edited with variant readings of the 1st and 2 nd editions and with a critical introduction and notes by F. E. L. Priestley, Toronto, University of Toronto Press, 1969. Désormais : Political Justice (PJ), selon le titre de 1793.

4 Il existe désormais une traduction française intégrale de Political Justice, postérieure à la rédaction de cet article : Enquête sur la justice politique, et son influence sur la morale et le bonheur d'aujourd'hui, trad. Denise Berthaud et Alain Thévenet, Lyon, Atelier de Création Libertaire, 2005. La précédente traduction était celle de Benjamin Constant, traduction incomplète et remaniée de la version de 1796 : De la justice politique, trad. inédite de l'ouvrage de W. Godwin par B. Constant, éd. B. R. Pollin, Québec, Presses de l'Université Laval, 1972. Disponible également dans les Euvres complètes de Constant, t. 2, Tübingen, Niemeyer, 1999.

5 Halévy, La formation, t. 2, p. 86 : «En dépit des différences, Godwin et Condorcet se trouvent avoir au moins une idée en commun : l'idée de la perfectibilité indéfinie de l'espèce humaine».

6 Reproduit dans Kegan Paul, William Godwin, His Friends and Contemporaries, 2 vol., Londres, $\begin{array}{lllll}\text { King, } & 1876, & \text { vol. } & 1, & \text { p. }\end{array}$

7 Pour une analyse de la notion de «modération», voir Bertrand Binoche, Introduction à De l'Esprit des lois de Montesquieu, Paris, Presses Universitaires de France, 1998, ch. 7, p. 243-295.

8 Things as They Are, or The Adventures of Caleb Williams [1794], préface à la deuxième édition, Londres, G. G. et J. Robinson, 1796, vol. 1, p. v. Traductions françaises disponibles : Germain Garnier, Paris, Agasse Librairie, 1795; Amédée Pichot [1868, $2^{\mathrm{e}}$ éd.], réimp. éd. Henri Veyrier, 1979; une traduction anonyme du XIX ${ }^{\mathrm{e}}$ siècle, Paris, Phébus, 1997.

9 Nous avons tenté de le démontrer dans «Roman de l'honneur et philosophie politique : Les choses comme elles sont, ou Les aventures de Caleb Williams de William Godwin (1794)», dans Colas Duflo et Luc Ruiz, dir., De Rabelais à Sade. L'Analyse des passions dans le roman de l'âge classique, Saint-Étienne, Publications de l'Université de Saint-Étienne, 2003.

10 Reproduit dans Kegan Paul, William Godwin, t. 2 p. 52.

11 Political Justice, après avoir connu un grand succès, retombe dans l'oubli dès le début du $\mathrm{XIX}^{\mathrm{e}}$ siècle : il est possible qu'il ait été lu comme une œuvre pamphlétaire de 
circonstance, périmée une fois que la volonté de réforme parlementaire est étouffée par le pouvoir ou qu'elle change de modèle, la France devenant l'ennemie d'une Angleterre en guerre, et sa révolution

ayant

été

trop

sanglante.

12 Voir l'article «ÉQUILIBRE(MÉCANIQUE)» de l'Encyclopédie, par D'Alembert, dans Encyclopédie, ou Dictionnaire raisonné des sciences, des arts et des métiers [1755], facsimilé, $\begin{array}{llllll}\text { Stuttgart, } & \text { Fromann, } & \text { 1966, } & \text { t. } & 5, & \text { p. }\end{array}$

13 William Godwin, Thoughts on Man, His Nature, Productions and Discoveries, Londres, Effingham Wilson, 1831, facsimile New York, Kelley, 1969. Voir, Essay II «Of Intellectual Abortion»: «We cannot stand still. If we do not go forward, we shall inevitably recede.»

14 Helvétius, De l'Esprit, éd. J. Moutaux, Paris, Fayard, 1988; désormais DE.

15 L'argument est un véritable topos au XVIII ${ }^{\mathrm{e}}$ siècle, voir Dix-huitième siècle, vol. 26. Sur son usage plus politique que démographique, J.-C. Perrot, Une histoire intellectuelle de l'économie politique (XVII ${ }^{e}$-XVIII ${ }^{e}$ siècle), Paris, Éditions des Hautes Etudes en Sciences Sociales, 1992, p. 161 : «la dépopulation est une arme des Lumières philosophiques».

16 Helvétius fait en effet une distinction entre la «loi suprême» du salut public qui est invariable; les «lois invariables», au nombre de quatre ou cinq (De l'Homme, éd. J. et G. Moutaux, 2 vol., Paris, Fayard, 1989, VII, note 7, p. 647-648), qui établissent la liberté et la propriété, c'est-à-dire les conventions fondatrices, et peuvent prendre plusieurs contenus mais poursuivent toutes le même but; et la majeure partie des lois, «variables», soit par elles-mêmes (elles s'adaptent aux circonstances), soit en raison de notre faible degré d'avancement dans la science de la législation. De l'Homme, X, 7, p. 907 sq. Désormais $D H$.

17 Rousseau, Émile ou De l'éducation, Paris, Gallimard, 1990.

18 On citera seulement, pour être bref, sur la matière : «On n'a point encore d'idées nettes et complètes de la matière», Helvétius, $D H$, IV, note 80 , p. 449; une définition possible de l'esprit comme «l'effet de la faculté de penser (et l'esprit n'est en ce sens que l'assemblage des pensées d'un homme)», $D E$, p. 15. Enfin, «ce que j'ai à dire de l'Esprit, s'accorde également bien avec l'une et l'autre» des hypothèses matérialiste et spiritualiste, $D E, \mathrm{I}, 1, \mathrm{p} .18$. La place manque ici mais on pourrait montrer que la décision épistémologique d'abandonner la perspective substantialiste comme philosophiquement indécidable, si ce n'est dans le langage des probabilités, s'appuie chez Helvétius sur la lecture de Berkeley, que Godwin cite explicitement.

19 PJ, t. 2, note p. 129-130 : «In his writings expressly political, Du Contrat Social and Considérations sur la Pologne, the superiority of his genius seems to desert him.»

20 Voir l'essai inédit «Of History and Romance», 1797, dans The Political and Philosophical Writings of William Godwin, general editor M. Philp, Londres, Pickering, 1993, vol. 5. 
21 H. Roussin, William Godwin, Paris, Plon-Nourrit, 1913, p. 168-169. Cité par F. E. L. Priestley,

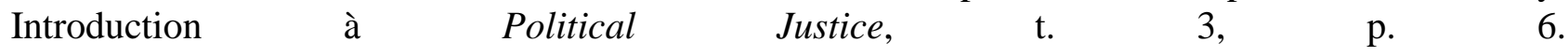

22 Une telle «contradiction» est un écho direct de L'Esprit des lois, IV, 4.

23 Bertrand Binoche, «William Godwin ou la véritable euthanasie des lois et des mœurs», Cahiers philosophiques de Strasbourg, 2002, vol. 12, p. 185-202, ici p. 198.

24 Lorsqu'il veut la reprendre à son compte, James Mill reconnaît que seul Helvétius défend l'égalité naturelle des esprits, mais «Helvétius à lui seul est une armée», dans l'article Éducation du supplément de l'Encyclopédie britannique, 1818, p. 18-20. Cité par Halévy, La formation, t. 2, p. 163.

25 William Godwin, The Enquirer : Reflections on Education, Manners and Literature in a Series of Essays, Londres, G. G. and J. Robinson, 1797, facsimile, New York, Kelley, 1965.

26 Pour une référence de Godwin ici, voir Priestley, Essay on the First Principles of Government, 1768. Je remercie Bertrand Binoche pour cette indication.

27 Esquisse d'un tableau historique des progrès de l'esprit humain, Paris, Flammarion, 1988, p. 231 : «on y vit se développer une doctrine nouvelle qui devait porter le dernier coup à l'édifice chancelant des préjugés : c'est celle de la perfectibilité indéfinie de l'espèce humaine, doctrine dont Turgot, Price et Priestley ont été les premiers et les plus illustres apôtres; elle appartient à la dixième époque

28 «Lettre sur un passage de la Décade philosophique et en général sur la perfectibilité de l'esprit humain», Euvres philosophiques, Paris, Presses universitaires de France, 1956, t. 2, p. 515, publiée dans la Décade du 19 avril 1799, citée par FrançoisAzouvi, «L'Institut National: une "encyclopédie vivante"?», dans François Azouvi, dir., L'institution de la raison, Paris, Vrin-EHESS, 1992, p. 5161. 